\title{
ČECH EXTENSIONS OF CONTRAVARIANT FUNCTORS $\left({ }^{1}\right)$
}

\author{
BY \\ C. N. LEE( $\left.{ }^{2}\right)$ AND FRANK RAYMOND( $\left(^{3}\right)$
}

Introduction. This paper studies and characterizes the Čech and singular methods of extending a set-valued functor from a category of triangulable pairs and homotopy classes of maps to a set-valued functor defined on more general topological pairs and homotopy classes of maps. Many new contravariant functors such as those in [1], [2], [4], [5], [6], [12], [15], have been introduced into algebraic topology. Their study has been responsible for much of the progress in the subject during recent years. They have been most successfully treated on a category of $C W$ complexes. It is natural to ask how one may extend these functors to a larger class of spaces, and to what extent does an extended functor possess properties similar to those enjoyed by the original functor defined on the smaller category. Furthermore, one should compare the possible extensions to determine if some have easily recognized properties which distinguish them from other possible extensions.

In classical cohomology theory, there are two standard methods, namely, those that yield the singular theory and the Čech theory. Both theories are defined on the largest class of spaces and yet satisfy all seven axioms postulated by Eilenberg and Steenrod [7]. For general spaces it often seems that the Čech theory is more preferable, whereas for homology, the singular theory is usually the only satisfactory one. (We feel that Theorem 2 and Appendix 1 partly "explain" this phenomenon and indicate that a similar statement may be applicable to generalized homology and cohomology theories.)

Let $\mathscr{C}$ be a category of topological pairs and continuous maps, and let $\mathscr{K}$ be a full subcategory of $\mathscr{C}$ whose objects are triangulable pairs such that each open covering of a pair in $\mathscr{C}$ has a refinement whose nerve is a pair in $\mathscr{K}$. We call the pair $(\mathscr{C}, \mathscr{K})$ a Čech extension category (see $\S 1$ for a more precise statement). Given a set-valued (contravariant) functor $F$ on $\mathscr{K}$, satisfying the homotopy axiom, we define the Čech functor $\breve{F}$ on $\mathscr{C}$, extending $F$. The bulk of this paper is devoted to examining which properties of the classical Čech cohomology theory are still valid for generalized Čech cohomology theories, and how $\check{F}$, as an extension of $F$, compares with other possible extensions. Many of the arguments, given by

(') Presented to the Society, October 29, 1966 under the title An extension of a generalized cohomology theory; received by the editors March 8, 1967 and, in revised form, April 28, 1967.

$\left({ }^{2}\right)$ Partial support from NSF grant 08105.

$\left({ }^{3}\right)$ Partial support from NSF grant 08105 and NSF Senior Faculty Fellowship. 
Eilenberg and Steenrod [7], for proofs of the axioms for the classical Čech cohomology theory carry over to our general Čech functor with little change.

The organization and an outline of our results are as follows: In $\S 1$, after setting up notation, we prove that $\not{F}$ satisfies the homotopy axiom, (Theorem 1 ). At this point we could pass to a category in which morphisms are homotopy classes of continuous maps instead of just continuous maps, but we found no essential advantage, and hence left the categories unaltered. In $\$ \$ 2$ through 4 , we require that the objects of $\mathscr{C}$ are paracompact Hausdorff pairs $(X, A)$ with $A$ closed in $X$. In $\S$, we define a natural transformation $\theta: \check{F} \rightarrow F$ on $\mathscr{C}$ where $F$ is also defined on $\mathscr{C}$ by assumption, that is, the initial $F$, defined on $\mathscr{K}$, is assumed to have been extended over $\mathscr{C}$. Let $F$ be homotopy representable by a topological pair $\left(E, E^{\prime}\right)$. Theorem 2 implies that $\theta: \check{F} \rightarrow F$ is an equivalence on $\mathscr{C}$ if and only if $\left(E, E^{\prime}\right)$ has the homotopy type of a $C W$-pair. This follows essentially from Theorem 1 and the definitions, thus differing from the conventional approach using the concept of "bridge maps" (Spanier [13], Hu [9], Barratt [3]). This theorem is used in $\$ 3$ to prove Theorem 3 which implies that the natural transformation $\theta: \check{F} \rightarrow F$ is equivalent (in some sense) to the canonical map: $\left(|S E|,\left|S E^{\prime}\right|\right) \rightarrow\left(E, E^{\prime}\right)$ where the domain pair is the geometric realization of the singular complex pair over $\left(E, E^{\prime}\right)$. In $\S 4$, we study the continuity axiom for $\check{F}$, and prove Theorem 5 asserting that $\check{F}$ is always weakly continuous. This seems to be the most general result known in this direction. This is used to prove that on certain categories $\theta: \breve{F} \rightarrow F$ is an equivalence if and only if $F$ is also weakly continuous (Theorems 4 and 5), where $F$ is assumed to have been defined on the category. In the last sections, we comment on the exactness axiom, excision axiom, and contravariant functors defined on a category in which objects are single spaces with base points.

In Appendix 1 we abstract the content of some of the paper and study the concept of a maximal and minimal extension of a contravariant or covariant setvalued functor. In this context the Čech extension of a contravariant (respectively, covariant) functor and the singular extension of a covariant (respectively, contravariant) functor are examples of unique maximal (respectively, minimal) extensions. The abstract form of Theorem 2 gives conditions for a representable extension to be equivalent to a maximal extension. Several topological applications, different from those treated in the text proper, are mentioned.

In Appendix 2 we formulate into a theorem the unique extendability of a functor (covariant or contravariant) defined on a subcategory $\mathscr{C}_{0}$ of a category $\mathscr{C}$, to a functor on the subcategory, $\mathscr{D}\left(\mathscr{C}_{0}\right)$, of those objects in $\mathscr{C}$ dominated by objects in $\mathscr{C}_{0}$.

Although our individual theorems, corollaries, and topological applications are new, at least stronger than the known ones to various degrees, our emphasis is not one or two main theorems, but all our results are produced here to answer the original questions stated in the first paragraph of this introduction. We intend to publish in the near future other aspects of generalized (singular) homology and 
(Čech) cohomology theories (for general spaces) including a Cartan-Leray type of spectral sequence of a map for homology and cohomology.

In the present paper, there is a small overlap with independent and unpublished results of A. Dold. In particular, he has also identified maximal extensions with the Čech extension and obtained the implication (iii) $\Rightarrow$ (ii) of Theorem 2.

\section{Extension of contravariant functors by the Cech method.}

1.1. Recall from Eilenberg-Steenrod [7, Chapter IX] the definitions of the family of coverings, $\operatorname{Cov}(X, A)$ of any topological pair $(X, A)$ and the nerve, $\left(X_{\alpha}, A_{\alpha}\right)$ of $\alpha \in \operatorname{Cov}(X, A)$. We shall use the same notation $\left(X_{\alpha}, A_{\alpha}\right)$ for its geometric realization $\left(\left|X_{\alpha}\right|,\left|A_{\alpha}\right|\right)$ with the weak topology. If $\mathscr{K}$ is a family of triangulable pairs, we shall denote by $\operatorname{Cov}^{\mathscr{X}}(X, A)$ the family of all $\alpha \in \operatorname{Cov}(X, A)$ such that $\left(X_{\alpha}, A_{\alpha}\right) \in \mathscr{K}$. Consider a pair $(\mathscr{C}, \mathscr{K})$ consisting of a category $\mathscr{C}$ of topological pairs and continuous maps between such pairs, and a full subcategory $\mathscr{K}$ of $\mathscr{C}$ whose objects are triangulable pairs. The pair $(\mathscr{C}, \mathscr{K})$ is called a Čech extension category if $\operatorname{Cov}^{\mathscr{X}}(X, A)$ is cofinal in $\operatorname{Cov}(X, A)$ for each pair $(X, A) \in \mathscr{C}$. For practical purposes, we shall further require that $\mathscr{K}$ and $\mathscr{C}$ are admissible categories for homology theory in the sense of Eilenberg-Steenrod [7, Chapter I], and that if $\left(K_{1}, L_{1}\right)$ is a subcomplex pair of a triangulable pair $(K, L) \in \mathscr{K}$ then $\left(K_{1}, L_{1}\right) \in \mathscr{K}$. Here again we identify a simplicial pair with its geometric realization. We shall in what follows continue to do this.

Some useful examples are:

$\left(\mathscr{C}_{c}, \mathscr{K}_{f}\right)$ where $\mathscr{C}_{c}=\{$ compact Hausdorff pairs and maps $\}$, and $\mathscr{K}_{f}=\{$ finitely triangulable pairs and maps $\}$,

$\left(\mathscr{C}_{s m}, \mathscr{K}_{l f}\right)$ where $\mathscr{C}_{s m}=\{$ separable metric pairs, and maps $\}$, and $\mathscr{K}_{l f}=\{$ countable locally finitely triangulable pairs, and maps\},

$\left(\mathscr{C}_{f d}, \mathscr{K}_{f d}\right)$ where $\mathscr{C}_{f d}=$ \{paracompact Hausdorff finite dimensional pairs, and maps $\}$, and $\mathscr{K}_{f d}=$ \{finite dimensional triangulable pairs, and maps $\}$,

$\left(\mathscr{C}_{1}, \mathscr{K}_{1}\right)$ where $\mathscr{C}_{1}=\{$ topological pairs and maps $\}$, and $\mathscr{K}_{1}=\{$ triangulable pairs and maps\},

$\left(\mathscr{C}_{p}, \mathscr{K}_{1}\right)$ where $\mathscr{C}_{p}=$ closed paracompact Hausdorff pairs, and maps $\}$ and $\mathscr{K}_{1}$ is the same as above. $(X, A) \in \mathscr{C}_{p}$ is a closed pair if $A$ is a closed subset of $X$.

1.2. Let $\mathscr{C}$ denote a category of topological pairs and continuous maps, and let $\mathscr{S}$ denote the category of sets and set-maps. Let $F: \mathscr{C} \rightarrow \mathscr{S}$ be a contravariant functor. $F$ is said to satisfy the homotopy axiom if $f$ and $g$ are maps in $\mathscr{C}$ which are homotopic to each other, $f \cong g$ by notation, then $F(f)=F(g)$.

Let $(\mathscr{C}, \mathscr{K})$ be a Čech extension category and let $F: \mathscr{K} \rightarrow \mathscr{S}$ be a contravariant functor satisfying the homotopy axiom. We shall define the associated Čech functor $\check{F}: \mathscr{C} \rightarrow \mathscr{S}$ such that $\breve{F} \mid \mathscr{K}$ is equivalent to $F$. (Once this has been done, one can easily modify the definition of $\check{F}$ so that the resulting functor is strictly equal to $F$ when restricted to. $\mathscr{K}$. This was pointed out to us by J. Schafer.) In order to avoid unnecessary complication, we shall be only concerned with $\breve{F}$, and regard 
this as an extension of $F$. To define $\mathscr{F}$, let $(X, A) \in \mathscr{C}$. For each $\alpha \in \operatorname{Cov}^{\mathscr{X}}(X, A)$, $F\left(X_{\alpha}, A_{\alpha}\right)$ is well defined. If $\alpha$ and $\beta \in \operatorname{Cov}^{\mathscr{X}}(X, A)$, and if $\beta$ refines $\alpha(\beta>\alpha$ by notation) then there is a map $\pi_{\alpha \beta}:\left(X_{\beta}, A_{\beta}\right) \rightarrow\left(X_{\alpha}, A_{\alpha}\right)$ called a projection map induced by the refining relation " $>$ ". Since any two projection maps are necessarily homotopic, the map $F\left(\pi_{\alpha \beta}\right): F\left(X_{\alpha}, A_{\alpha}\right) \rightarrow F\left(X_{\beta}, A_{\beta}\right)$ does not depend on the choice of projection map, and hence $F\left(\pi_{\alpha \beta}\right)$ is uniquely defined by the refining relation $\beta>\alpha$. It follows that the family of sets $F\left(X_{\alpha}, A_{\alpha}\right)$, for $\alpha \in \operatorname{Cov}^{\mathscr{K}}(X, A)$, and maps $F\left(\pi_{\alpha \beta}\right)$ form a direct system. We define

$$
\check{F}(X, A)=\operatorname{Dir} \operatorname{Lim}\left\{F\left(X_{\alpha}, A_{\alpha}\right) ; F\left(\pi_{\alpha \beta}\right)\right\}
$$

where the direct limit is taken over the direct system just described. To complete the definition of $\breve{F}$, we must define $\breve{F}$ on maps. Let $f:(X, A) \rightarrow(Y, B)$ be in $\mathscr{C}$. For any $\alpha \in \operatorname{Cov}^{\mathscr{X}}(Y, B), \alpha^{\prime}=f^{-1}(\alpha) \in \operatorname{Cov}^{\mathscr{X}}(X, A)$ because $\left(X_{\alpha^{\prime}}, A_{\alpha^{\prime}}\right)$ is a subcomplex pair of $\left(Y_{\alpha}, B_{\alpha}\right)$ and hence belongs to $\mathscr{K}$ by the definition of $\mathscr{K}$. Thus, the inclusion map $i_{\alpha}:\left(X_{\alpha^{\prime}}, A_{\alpha^{\prime}}\right) \rightarrow\left(Y_{\alpha}, B_{\alpha}\right)$ is in $\mathscr{K}$. In particular, $F\left(i_{\alpha}\right)$ is defined. Furthermore, the map: $\operatorname{Cov}^{\mathscr{}}(Y, B) \rightarrow \operatorname{Cov}^{\mathscr{}}(X, A)$ induced by $f$ preserves the refining relation, and hence we may define the map $\breve{F}(f): \check{F}(Y, B) \rightarrow \check{F}(X, A)$ by passing to the limit. More details may be obtained by modifying the contents of $\$ \$ 2,3$ and 4 of [7, IX]. This completes the definition of the contravariant functor $\breve{F}: \mathscr{C} \rightarrow \mathscr{S}$ called the Cech extension of the contravariant functor $F: \mathscr{K} \rightarrow \mathscr{S}$. The word "extension" will be justified in the next section. We shall first prove

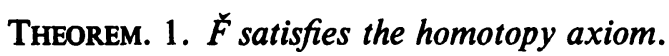

This is proved for the ordinary Čech cohomology theory in [7]. To prove it in the general case as stated above, one needs only follow the same argument with slight alterations at some steps. We shall indicate these alterations and leave the details to the reader.

First, take Lemmas 5.2, 5.4, 5.6, 5.7 and 5.8 of [7, pp. 240-242], and alter the statements of Lemmas 5.2 and 5.7 by replacing the word "acyclic" by "contractible". The proofs of these altered statements are similar to the original ones. Also, change in Lemma 5.8 the assertion to read " $l$ is homotopic to $u$ ". The altered part of this proof is as follows: For each simplex $s$ of the nerve $X_{\alpha}$ (considered as a simplicial complex), let $C(s)$ be the subcomplex of $(X \times I)_{y}$ consisting of all the simplices whose vertices have the form $(v, i)$ where $v$ is a vertex of $s$. The simplex $s$ is the nerve of a covering $\alpha^{\prime}$ of a subset $X^{\prime}$ of $X$ and $C(s)$ is contractible, by the alteration of Lemma 5.7 of [7]. If $s^{\prime}$ is a face of $s$ then $C\left(s^{\prime}\right) \subset C(s)$; and if $s$ is in $A_{\alpha}$, then $C(s)$ is in $(A \times I)_{y}$. Note that $l(v)=(v, 0)$ and $u(v)=\left(v, n^{v}\right)$ for any vertex $v$ of $X_{\alpha}$. Define a homotopy $k:\left(X_{\alpha} \times I\right) \rightarrow(X \times I)_{\gamma}$ by an induction on the skeleta of $X_{\alpha}$ such that $k\left(s^{n} \times I\right) \subset C\left(s^{n}\right)$ for each $n$-simplex $s^{n}$ of $X_{\alpha}$ with $k_{0}=l$ and $k_{1}=u$. The remainder of the argument is the same as that given on p. 243 of [7]. Note in carrying out this argument there arises a small difficulty from the fact that the 
nerve of a stacked covering of $X \times I$ may fail to be an object in $\mathscr{K}$. However, the conditions defining the Čech extension category $(\mathscr{C}, \mathscr{K})$ enable one to get around this minor difficulty.

1.4. Remarks. 1. We may obtain analogous results for covariant functors with the same argument.

2. Recall the notation $\left(\mathscr{C}_{c}, \mathscr{K}_{f}\right)$ in 1.1 . It is possible to define a Čech extension of a (either covariant or contravariant) functor $F: \mathscr{K}_{f} \rightarrow \mathscr{S}$ having the property that if $f, g:(X, A) \rightarrow(Y, B)$ are maps in $\mathscr{K}_{f}$ which are contiguous with respect to some triangulations of the pairs then $F(f)=F(g)$. In proving that the resulting Čech extension $\breve{F}$ satisfies the homotopy axiom, one needs only replace "acyclic" by "collapsible" instead of "contractible".

3. Denote by $\mathscr{S}^{\mathscr{X}}$ the category of contravariant functors: $\mathscr{K} \rightarrow \mathscr{S}$ satisfying the homotopy axiom as objects and natural transformations between such functors as morphisms. Similarly for $\mathscr{S}^{\mathscr{C}}$. These categories are not "legitimate" since the natural transformations from one functor into another may not form a set. For each $F \in \mathscr{S}^{\mathscr{X}}, \check{F} \in \mathscr{S}^{\mathscr{C}}$ since $\breve{F}$ also satisfies the homotopy axiom by Theorem 1 . Write Čech $(F)=\breve{F}$ for each $F \in \mathscr{S}^{\mathscr{X}}$. If $\tau: F \rightarrow G$ is a morphism in $\mathscr{S}^{\mathscr{X}}$ then Čech $(\tau)=\check{\tau}: \check{F} \rightarrow \breve{G}$ is well defined in the obvious manner. It is easy to check that we have a (covariant) functor Čech: $\mathscr{S}^{\mathscr{X}} \rightarrow \mathscr{S}^{\mathscr{C}}$. We have another covariant functor Res: $\mathscr{S}^{\mathscr{C}} \rightarrow \mathscr{S}^{\mathscr{X}}$ defined by restriction. It will follow from 2.2 that the composite functor Res。Cech is equivalent to the identity functor on $\mathscr{S} \mathscr{X}$.

\section{Comparison of the Cech extension with an arbitrary extension.}

2.1. Let $(\mathscr{C}, \mathscr{K})$ be a Čech extension category as defined in $\S 1$. Note that $(\mathscr{K}, \mathscr{K})$ is also a Čech extension category. We shall assume in this section that the objects of $\mathscr{C}$ are paracompact Hausdorff closed pairs. (By a closed pair $(X, A)$, we mean $A$ is a closed subspace of $X$ in which case $\operatorname{Cov}(X, A)$ needs only be singly indexed [7, p. 249].) Let $F: \mathscr{C} \rightarrow \mathscr{S}$ be a contravariant functor satisfying the homotopy axiom, and let $\breve{F}: \mathscr{C} \rightarrow \mathscr{S}$ be the Čech extension of $F \mid \mathscr{K}$. We shall define a natural transformation $\theta: \breve{F} \rightarrow F$.

Let $(X, A) \in \mathscr{C}$ and $\alpha \in \operatorname{Cov}(X, A)$. If $\alpha$ is locally finite then there is a continuous map $\varphi_{\alpha}:(X, A) \rightarrow\left(X_{\alpha}, A_{\alpha}\right)$ called a barycentric (or canonical) map. To be more specific, let $\left\{\lambda_{i} \mid i \in V_{\alpha}\right\}$ be a partition of unity subordinated to $\alpha$. Then $\varphi(x)$ is defined to be the point of the simplicial complex $X_{\alpha}$ with the barycentric coordinate $\left\{\lambda_{i}(x) \mid i \in V_{\alpha}\right\}$. For $\alpha \in \operatorname{Cov}(X, A)$, $\alpha$ not necessarily locally finite, we find a locally finite $\beta, \beta>\alpha$, and simply define $\varphi_{\alpha}=\pi_{\alpha \beta} \varphi_{\beta}$. It is easy to prove that these maps are well defined up to homotopy. As a consequence of this, we get a well-defined $\operatorname{map} \theta_{X, A}: \check{F}(X, A) \rightarrow F(X, A)$ by $\left\{F\left(\varphi_{\alpha}\right) \mid \alpha \in \operatorname{Cov}^{\mathscr{X}}(X, A)\right\}$.

We have already observed that if $\mathscr{K}$ is an admissible category of triangulable pairs, then $(\mathscr{K}, \mathscr{K})$ is a Čech extension category. The next proposition states, in particular, that if $F: \mathscr{K} \rightarrow \mathscr{S}$ is as in $\S 1$, then $\breve{F}$ is an "extension" of $F$ and that $\theta$ is the natural transformation which induces this extension. 
2.2. Proposition. $\theta$ is a natural transformation. Furthermore, $\theta \mid \mathscr{K}$ is an equivalence representing $\breve{F}$ as an extension of $F \mid \mathscr{K}$.

Proof. The first assertion follows from Theorem 1 and the definitions. It is easily checked. To prove the next assertion, let $(X, A)$ be a pair in $\mathscr{K}$ and consider the commutative diagram

$$
\begin{gathered}
\left(X_{\alpha}, A_{\alpha}\right) \stackrel{\varphi_{\alpha}=1}{\longleftarrow}(|K|,|L|) \\
\uparrow 1 \simeq \pi_{\alpha \beta} \uparrow_{1}^{\longleftarrow}\left(\left|K^{\prime}\right|,\left|L^{\prime}\right|\right)
\end{gathered}
$$

where $(K, L)$ is a triangulation of $(X, A)$ and $\left(K^{\prime}, L^{\prime}\right)$ is a subdivision (not necessarily barycentric) of $(K, L)$, and $\alpha$ (resp., $\beta)$ is the open covering consisting of open stars of the vertices of $K$ (resp., $\left.K^{\prime}\right)$. Note that $(K, L)$ and hence $\left(K^{\prime}, L^{\prime}\right)$ may not be locally finite, but the identity maps $\varphi_{\alpha}$ and $\varphi_{\beta}$ are clearly continuous and are homotopic to barycentric maps. We can obtain a cofinal family of such coverings of $(X, A)$. We have already observed that if $\gamma \in \operatorname{Cov}(X, A)$ and $\gamma>\alpha$ then $\pi_{\alpha \gamma} \varphi_{\gamma}$ $\cong \varphi_{\alpha}$. The assertion now follows.

For each $\alpha \in \operatorname{Cov}^{\mathscr{X}}(X, A)$, each element $a_{\alpha} \in F\left(X_{\alpha}, A_{\alpha}\right)$ represents a unique element $\check{a}=p_{\alpha}\left(a_{\alpha}\right) \in \breve{F}(X, A)$. Here the $p_{\alpha}$ are those maps representing $\breve{F}(X, A)$ as the direct limit of the $F\left(X_{\alpha}, A_{\alpha}\right), \alpha \in \operatorname{Cov}^{\mathscr{X}}(X, A)$.

Corollary. If $(X, A)=(|K|,|L|) \in \mathscr{K}$ and $\alpha$ is the covering by open stars of the vertices of $K$ then the following maps are inverses to each other:

$$
\check{F}(X, A) \underset{p_{\alpha}}{\stackrel{\theta_{X, A}}{\rightleftarrows}} F\left(X_{\alpha}, A_{\alpha}\right) .
$$

We remark that if we make the natural identification of the triangulable pair with the geometric realization of the nerve of the covering formed by the open stars of the vertices of a triangulation of $(X, A)$, then $\theta_{X, A}$ is the identity map. Furthermore, in terms of these identifications, $F(f)=\breve{F}(f)$, for $f:(X, A) \rightarrow(Y, B)$, where $(X, A)$ and $(Y, B) \in \mathscr{K}$.

2.3. Remark. $\theta$ is defined for each $F \in \mathscr{S}^{\mathscr{X}}$ so that $\theta_{F}: \breve{F} \rightarrow F$ is a natural transformation. In fact, such assignment $\theta: F \rightarrow \theta_{F}$ is natural with respect to morphisms in $\mathscr{S}^{x}$ in the sense that if $\tau: F \rightarrow G$ is a morphism in $\mathscr{S}^{x}$ then we have the commutative diagram

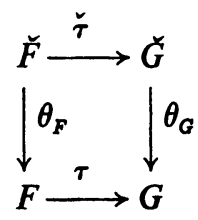


2.4. LeMmA. If $a_{\alpha} \in F\left(X_{\alpha}, A_{\alpha}\right)$ represents $\check{a} \in \check{F}(X, A)$ where $\alpha \in \operatorname{Cov}^{\mathscr{K}}(X, A)$ then

$$
\check{F}\left(\varphi_{\alpha}\right) \theta_{\bar{x}_{\alpha}, A_{\alpha}}^{-1}\left(a_{\alpha}\right)=\check{a} .
$$

Proof. Denote by the same $\alpha$ the covering of $\left(X_{\alpha}, A_{\alpha}\right)$ by open stars of the vertices of $X_{\alpha}$, and by the same $\left(X_{\alpha}, A_{\alpha}\right)$ its nerve. Consider the diagram

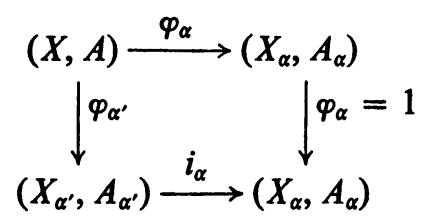

where $\alpha^{\prime}=\varphi_{\alpha}^{-1}(\alpha)$ and $i_{\alpha}$ is the inclusion map. Since $\alpha^{\prime}$ refines $\alpha, i_{\alpha}$ is a projection map $\pi_{\alpha \alpha^{\prime}}$. Hence, in the induced diagram

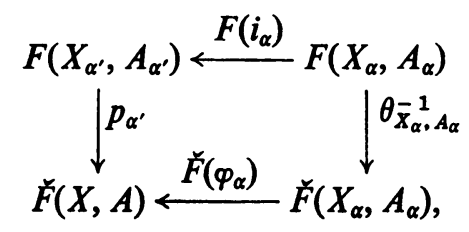

we get $p_{\alpha}=p_{\alpha^{\prime}} F\left(i_{\alpha}\right)$ where the maps $p_{\alpha}, p_{\alpha^{\prime}}$ were defined in 2.2 . We now need only show that the diagram commutes, but this follows from the definition of $\breve{F}\left(\varphi_{\alpha}\right)$ and the corollary in 2.2 .

2.5. Let $F_{1}$ and $F_{2}: \mathscr{C} \rightarrow \mathscr{S}$ be contravariant functors. Assume that $F_{2}$ is homotopy-representable by a pair $\left(E, E^{\prime}\right) \in \mathscr{C}$, i.e., $F_{2}$ is equivalent to the functor [ $; E, E^{\prime}$ ], the homotopy classes of the maps of pairs of $\mathscr{C}$ into $\left(E, E^{\prime}\right)$. For simplicity, let $F_{2}=\left[; E, E^{\prime}\right]$. Given any $a \in F_{1}\left(E, E^{\prime}\right)$ there is a natural transformation $\psi^{a}: F_{2} \rightarrow F_{1}$ defined by $\psi_{X, A}^{a}(f)=F_{1}(f)(a)$ for $f \in F_{2}(X, A)$. In fact, every natural transformation from $F_{2}$ to $F_{1}$ is obtained this way.

LEMMA. Suppose that there is a natural transformation $\theta: F_{1} \rightarrow F_{2}$ and an element $e \in F_{1}\left(E, E^{\prime}\right)$ with $\theta_{E, E^{\prime}}(e) \cong 1:\left(E, E^{\prime}\right) \rightarrow\left(E, E^{\prime}\right)$. Then, there exists a natural transformation $\psi: F_{2} \rightarrow F_{1}$ such that $\theta \psi=1$, the identity transformation of $F_{2}$ onto itself.

Proof. Simply let $\psi=\psi^{e}$.

2.6. Preserving the notation of 2.1, assume further that $F$ is homotopyrepresentable by $\left(E, E^{\prime}\right) \in \mathscr{C}$ and that for some $e \in \check{F}\left(E, E^{\prime}\right), \theta_{E, E^{\prime}}(e) \cong 1$.

Lemma. For $\check{a} \in \check{F}(X, A)$ and $a_{\alpha} \in F\left(X_{\alpha}, A_{\alpha}\right)$ representing $\check{a}$, where $\alpha \in \operatorname{Cov}^{\mathscr{X}}(X, A)$ we have

$$
\check{F}\left(\varphi_{\alpha}\right) \psi_{X_{\alpha}, A_{\alpha}}^{e}\left(a_{\alpha}\right)=\check{a} .
$$

In particular, $\psi_{X, A}^{e}$ is a bijection. 
Proof. Note $\theta_{\bar{x}_{\alpha}, A_{\alpha}}^{-1}\left(a_{\alpha}\right)=\psi_{X_{\alpha}, A_{\alpha}}^{e}\left(a_{\alpha}\right)$ by 2.2 and 2.5. By substituting in 2.4, we obtain the desired formula above. Because $\theta \psi=1$, by $2.5, \psi_{X, A}^{e}$ is injective. Since $\check{a}=\check{F}\left(\varphi_{\alpha}\right) \psi_{X_{\alpha}, A_{\alpha}}^{e}\left(a_{\alpha}\right)=\psi_{X, A}^{e} F\left(\varphi_{\alpha}\right)\left(a_{\alpha}\right), \psi_{X, A}^{e}$ is also surjective.

2.7. Let $(\mathscr{C}, \mathscr{K})$ be as in 2.1 where the objects of $\mathscr{C}$ are by assumption paracompact Hausdorff closed pairs, and let $F: \mathscr{C} \rightarrow \mathscr{S}$ be a contravariant functor which is homotopy-representable by $\left(E, E^{\prime}\right) \in \mathscr{C}$.

THEOREM 2. The following are equivalent:

(i) There exists $e \in \check{F}\left(E, E^{\prime}\right)$ such that $\theta_{E, E^{\prime}}(e)=1:\left(E, E^{\prime}\right) \rightarrow\left(E, E^{\prime}\right)$.

(ii) $\theta$ is a natural equivalence.

(iii) $\left(E, E^{\prime}\right)$ is dominated by a pair in $\mathscr{K}$.

(iv) $\left(E, E^{\prime}\right)$ has the homotopy type of a triangulable pair (not necessarily in $\mathscr{C}$ ).

(v) $\breve{F}$ is also homotopy-representable by $\left(E, E^{\prime}\right)$.

Proof. (i) $\Leftrightarrow$ (ii), follows from 2.6.

(ii) $\Rightarrow$ (iii). Let $e, e \in \breve{F}\left(E, E^{\prime}\right)$, be such that $\theta_{E, E^{\prime}}(e)=1 \in F\left(E, E^{\prime}\right)$. Let $e_{\alpha}, e_{\alpha} \in$ $F\left(X_{\alpha}, A_{\alpha}\right)$, represent $e$ where $\alpha \in \operatorname{Cov}^{\mathscr{X}}(X, A)$. By 2.6, $e=\check{F}\left(\varphi_{\alpha}\right) \theta_{E_{\alpha}, E_{\alpha}^{\prime}}^{1}\left(e_{\alpha}\right)$, and hence $\theta_{E, E^{\prime}}(e)=F\left(\varphi_{\alpha}\right)\left(e_{\alpha}\right)=1$. That is, there are maps

$$
\left(E_{\alpha}, E_{\alpha}^{\prime}\right) \underset{\varphi_{\alpha}}{\stackrel{e_{\alpha}}{\rightleftarrows}}\left(E, E^{\prime}\right)
$$

such that $e_{\alpha} \circ \varphi_{\alpha} \cong 1$. Thus $\left(E, E^{\prime}\right)$ is dominated by the triangulable pair $\left(E_{\alpha}, E_{\alpha}^{\prime}\right) \in \mathscr{K}$.

(iii) $\Rightarrow$ (iv). Since $\left(E, E^{\prime}\right)$ is dominated by a pair $\left(E_{\alpha}, E_{\alpha}^{\prime}\right) \in \mathscr{K},\left(E, E^{\prime}\right)$ has the homotopy type of a triangulable pair by a well known result of J. H. C. Whitehead.

(iii) $\Rightarrow$ (i). Let $(X, A) \in \mathscr{K}$ and $f, g$ be maps, $f:(X, A) \rightarrow\left(E, E^{\prime}\right), g:\left(E, E^{\prime}\right)$ $\rightarrow(X, A)$, such that $f \circ g \cong 1_{E, E^{\prime}}$. Let $[f]$ denote the homotopy class of $f$ and set $e=\breve{F}(g) \theta_{\bar{x}, A}^{-1}[f], e \in \check{F}\left(E, E^{\prime}\right)$. Now

$$
\theta_{E, E^{\prime}}(e)=\theta_{E, E^{\prime}} \breve{F}(g) \theta_{\bar{X}, A}^{-1}[f]=F(g)[f]=[f \circ g]=1_{E, E^{\prime}}
$$

(iv) $\Rightarrow$ (iii). The triangulable pair $(K, L)$ clearly dominates $\left(E, E^{\prime}\right)$ if $(K, L)$ and $\left(E, E^{\prime}\right)$ have the same homotopy type. However, $(K, L)$ may not be a pair in $\mathscr{K}$. To remedy this, consider the functor $\left[; E, E^{\prime}\right]$ defined on $\mathscr{C}_{p}$. It can be regarded as an extension of the same functor restricted to $\mathscr{K}_{1}$. The hypothesis of (iii) is satisfied for the Čech extension category $\left(\mathscr{C}_{p}, \mathscr{K}_{1}\right)$ and the homotopy-representable functor [ ; $\left.E, E^{\prime}\right]$. Thus the argument of (ii) $\Rightarrow$ (iii) shows that $\left(E, E^{\prime}\right)$ is dominated by a nerve $\left(E_{\alpha}, E_{\alpha}^{\prime}\right)$ of some covering $\alpha \in \operatorname{Cov}^{\mathscr{X}_{1}}\left(E, E^{\prime}\right)$. Since $\left(E, E^{\prime}\right) \in \mathscr{C}$, choose a covering $\beta, \beta \in \operatorname{Cov}^{\mathscr{X}}\left(E, E^{\prime}\right)$, and $\beta>\alpha$. If the domination of $\left(E, E^{\prime}\right)$ by $\left(E_{\alpha}, E_{\alpha}^{\prime}\right)$ is given by, $\left(E, E^{\prime}\right) \stackrel{\Phi_{\alpha}}{\longrightarrow}\left(E_{\alpha}, E_{\alpha}^{\prime}\right) \stackrel{e_{\alpha}}{\longrightarrow}\left(E, E^{\prime}\right)$, as in (ii) $\Rightarrow$ (iii), then the composition, $\left(E, E^{\prime}\right) \stackrel{\Phi_{\beta}}{\longrightarrow}\left(E_{\beta}, E_{\beta}^{\prime}\right) \stackrel{e_{\alpha} \circ \pi_{\alpha \beta}}{\longrightarrow}\left(E, E^{\prime}\right)$, yields a domination of $\left(E, E^{\prime}\right)$ by the nerve $\left(E_{\beta}, E_{\beta}^{\prime}\right), \beta \in \operatorname{Cov}^{\mathscr{X}}\left(E, E^{\prime}\right)$.

(v) $\Leftrightarrow$ (ii). This is clear, whereby we complete the proof. 
2.8. Corollary (Spanier [14], Hu [9], Barratt [3]). Let $(\mathscr{C}, \mathscr{K})$ be a Čech extension category and $\left(E, E^{\prime}\right)$ be a pair in $\mathscr{C}$ having the homotopy type of some $C W$-pair. Then, for any $(X, A) \in \mathscr{C}$,

$$
\operatorname{Dir}_{\alpha \in \operatorname{Cov}^{\mathcal{X}} \mathcal{L i m}_{(\mathrm{X}, A)}}\left[X_{\alpha}, A_{\alpha} ; E, E^{\prime}\right] \stackrel{\theta}{\approx}\left[X, A ; E, E^{\prime}\right] .
$$

This result is more general than the corresponding results in [3], [9], and [14], in that no restrictions on the $C W$-pair or on $(X, A) \in \mathscr{C}$ are imposed.

To illustrate this corollary, we mention (1) the representability of the ordinary Čech cohomology functor $\check{H}^{n}(X, A ; G)$ by $(K(G, n), p t)$ on $\mathscr{C}_{p}$ where $G$ is an abelian group, that is, $\check{H}^{n}(X, A ; G) \approx[X, A ; K(G, n), p t]$ (also proved by Huber in [10] but with mild restrictions either on $G$ or $(X, A)$ ), and similarly (2) if $(X, A) \in \mathscr{C}_{p}$ has relative covering dimension $\leqq n$, then $\breve{H}^{n}(X, A ; Z) \approx\left[X, A ; S^{n}, p t\right]$.

2.9. REMARK. We describe here very briefly another method of proving the Corollary in 2.8 following the classical techniques as used in [3], [9] and [14] where $\left(E, E^{\prime}\right)$ is assumed to be triangulated. Using the triangulation, one defines a socalled bridge map $f_{\alpha}:\left(X_{\alpha}, A_{\alpha}\right) \rightarrow\left(E, E^{\prime}\right)$ for a given map $f:(X, A) \rightarrow\left(E, E^{\prime}\right)$ such that $f_{\alpha} \cdot \varphi_{\alpha} \cong f$ where $\varphi_{\alpha}$ is a barycentric map. It is not difficult to prove that the map $\theta$ is onto. The difficult part is to prove that $\theta$ is injective. For this one needs to construct homotopic bridge maps $f_{\alpha} \cong g_{\alpha}$ for homotopic maps $f \cong g$.

3. The representability of the Cech extension. In practice, it often occurs that a contravariant functor: $\mathscr{K} \rightarrow \mathscr{S}$ is homotopy representable by an object which is not in $\mathscr{K}$ or any of the categories $\mathscr{C}$ associated with $\mathscr{K}$. For example, the Grothendieck group of complex vector bundles over finite dimensional paracompact Hausdorff spaces is homotopy. represented by $Z \times B_{U}$ but the classifying space $B_{U}$ is not finite dimensional. Thus Theorem 2 does not apply as (i) and (ii) have no meaning.

The following three examples are relevant to this discussion.

3.1. Let $\boldsymbol{F}$ be the functor [ $\left.; \boldsymbol{R}^{n}, \boldsymbol{R}^{n}-\{0\}\right]$ where $\boldsymbol{R}^{n}$ denotes euclidean $n$-space. When restricted to $\mathscr{K}_{f}, F$ is also equivalent to the functor [ ; $\left.D^{n}, D^{n}\right]$ where $D^{n}$ is the closed $n$-disk and $\dot{D}^{n}$ its boundary sphere. The associated Čech functor on $\mathscr{C}_{c}, \breve{F}$, is therefore homotopy-represented by $\left(D^{n}, \dot{D}^{n}\right)$. The natural transformation $\theta: \breve{F} \rightarrow F$ is an equivalence on $\mathscr{C}_{c}$. Of course, $\left(\boldsymbol{R}^{n}, \boldsymbol{R}^{n}-\{0\}\right)$ does not have the homotopy type of a $C W$-pair.

3.2. For each triangulable space $X$, define $F(X)=$ the inverse limit of $H^{2}\left(X_{\alpha} ; Z\right)$ where $X_{\alpha}$ runs through the set of all compact triangulable subspaces of $X$. This functor $F$ is homotopy represented by $K(Z, 2)$ on $\mathscr{X}_{\text {f }}$ but not homotopy representable by any space on the category of locally finite triangulable spaces.

3.3. Let $\left(E, E^{\prime}\right)$ be the pair consisting of the "Warsaw circle" $E$ and a base point $E^{\prime}=\{*\}$ where $E$ is the set of all points $(x, y) \in R^{2}$ satisfying $y=\sin 1 / x$, $0<x \leqq 2 / \pi$, or $-3 / 2 \leqq y<1, x=0$ or $2 / \pi$, or $y=-3 / 2,0 \leqq x \leqq 2 / \pi$, and $*=(0,1)$. Then the functor $F=\left[; E, E^{\prime}\right]$ is equivalent on $\mathscr{K}_{f}$ to the functor $[; p t, p t]$. It 
follows from 3.6 below that on $\mathscr{C}_{c}, \check{F}$ is also homotopy representable by ( $\left.p t, p t\right)$. The natural transformation $\theta$ is, of course, not an equivalence on $\mathscr{C}_{c}$, for example, $F\left(E, E^{\prime}\right)$ is infinitely generated whereas $\breve{F}\left(E, E^{\prime}\right)$ is a single element.

3.4. Let $F=\left[; E, E^{\prime}\right]$ defined on $\mathscr{C}_{p}$ where $\left(E, E^{\prime}\right)$ is any topological pair. Let $f:(K, L) \rightarrow\left(E, E^{\prime}\right)$ be a map where $(K, L)$ has the homotopy type of a triangulable pair such that all three maps, $f, f|K, f| L$ are singular homotopy equivalences. Such $(K, L)$ and $f$ always exist by taking, for example, the geometric realization ( $\left.|S E|,\left|S E^{\prime}\right|\right)$ of the singular complex pairs. Let $G=[; K, L]$ and $f_{\#}: G \rightarrow F$ be the induced natural transformation. Since the Čech process is natural, we have the commutative diagram

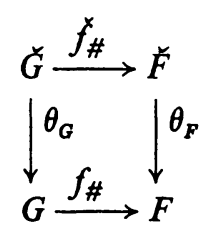

of functors and natural transformations. By generalizing [13, 7.6.23, p. 405] to pairs and passing to the direct limit, we see that $f_{\#}$ is an equivalence. Theorem 2 , on the other hand, implies that $\theta_{G}$ is an equivalence. In this sense, $f_{\#}$ is equivalent to $\theta: \check{F} \rightarrow F$. In particular, $\theta_{F}$ is an equivalence if and only if $f_{\#}$ is an equivalence on $\mathscr{C}_{p}$.

3.5. Let $(\mathscr{C}, \mathscr{K})$ be a Čech extension category as in 2.1 , and let $F: \check{\mathscr{K}} \rightarrow \mathscr{S}$ be a contravariant functor homotopy representable by a topological pair $\left(E, E^{\prime}\right)$ not necessarily in $\mathscr{C}$. Extend $F$ to $\mathscr{F}$ on $\mathscr{C}$. With the aid of $\hat{G}$ and $G$ of 3.4 , one deduces

THEOREM 3. $\check{F}$ is homotopy representable on $\mathscr{C}$ by a triangulable pair $(K, L)$ having the homotopy type of $\left(|S E|,\left|S E^{\prime}\right|\right)$. Furthermore, if $F$ was already the restriction of a functor, also denoted by $F$, on $\mathscr{C}$ and homotopy representable on $\mathscr{C}$ by $\left(E, E^{\prime}\right)$, then the natural transformation $\theta: \breve{F} \rightarrow F$ is equivalent to the natural transformation $f_{\#}:[; K, L] \rightarrow\left[; E, E^{\prime}\right]$ where $f:(K, L) \rightarrow\left(E, E^{\prime}\right)$ is a singular homotopy equivalence.

\section{The continuity of the Cech functor.}

4.1. Tentatively, let $\mathscr{C}$ be a fixed but unspecified category of topological pairs and continuous maps. Objects will always be taken from $\mathscr{C}$.

Let $\left\{\left(X_{m}, A_{m}\right), f_{n, m}\right\}$ be an inverse system where each $\left(X_{m}, A_{m}\right)$ are subspaces of a fixed space $Y$, and $f_{n, m}(n>m)$ is the inclusion map: $\left(X_{m}, A_{m}\right) \subset\left(X_{n}, A_{n}\right)$. We shall call such a system a nested system for $(X, A)$ in $Y$ if $(X, A)=\bigcap_{m \in M}\left(X_{m}, A_{m}\right)$ and each neighborhood pair $(U, V)$ of $(X, A)$ contains some $\left(X_{m}, A_{m}\right)$.

Let $F: \mathscr{C} \rightarrow \mathscr{S}$ be a contravariant functor. $F$ is said to be continuous (resp., weakly continuous) at $(X, A)$ in $\mathscr{C}$ if for any inverse (resp., nested) system $\left\{\left(X_{m}, A_{m}\right)\right.$, $\left.f_{n, m}\right\}$ whose limit is $(X, A)$ the canonical map $\mu: \operatorname{Dir} \operatorname{Lim} F\left(X_{m}, A_{m}\right) \rightarrow F(X, A)$ is bijective. If $F$ is continuous (resp., weakly continuous) at every $(X, A) \in \mathscr{C}$ then we shall simply say $F$ is continuous (resp., weakly continuous) in $\mathscr{C}$. 
4.2. Recall that $\left(\mathscr{C}_{c}, \mathscr{K}_{f}\right)$ denotes the Čech extension category where the objects of $\mathscr{C}_{c}$ (respectively, $\mathscr{K}_{f}$ ) are compact Hausdorff (respectively, finitely triangulable) pairs.

THEOREM 4. Let $F: \mathscr{C}_{c} \rightarrow \mathscr{S}$ be a contravariant functor satisfying the homotopy axiom. Then the following are equivalent:

(1) $F$ is continuous in $\mathscr{C}_{c}$.

(2) $F$ is weakly continuous in $\mathscr{C}_{c}$.

(3) The natural transformation $\theta: \check{F} \rightarrow F$ is an equivalence on $\mathscr{C}_{c}$ where $\check{F}$ is the Cech extension of $F \mid \mathscr{K}_{f}$.

We first state two well-known results which can be used in the proof.

4.3. Lemma. For any compact Hausdorff pair $(X, A)$, there exists a nested system in some compact Hausdorff space $Y$ such that each pair of the system has the homotopy type of a finitely triangulable pair.

4.4. Lemma. For each inverse system $\left\{\left(X_{n}, A_{n}\right), f_{n, m}\right\}$ of compact Hausdorff pairs the inverse limit $(X, A)$ can be imbedded in a space $Y$ in which it is the limit of a nested system of compact Hausdorff pairs $\left\{\left(X_{n}^{\prime}, A_{n}^{\prime}\right)\right\}$, with the same indexing set, and where each $\left(X_{n}^{\prime}, A_{n}^{\prime}\right)$ has the same homotopy type as $\left(X_{n}, A_{n}\right)$.

For a proof of 4.3 see $[13$, p. 319] or [7, pp. 284-285]. For 4.4 see [13, p. 358, C-1] or [7, pp. 293-294, A1-3].

For the proof of the theorem it is clear that $(1) \Rightarrow(2)$. By Theorem 5 , which will be proved independently, $\breve{F}$ is also weakly continuous and hence $(2) \Rightarrow(3)$ is an easy consequence of 4.3. By an exact analogue to the proof of continuity for ordinary Čech cohomology theory as given in [7, Chapter $X, \S 3]$, we can prove that $\check{F}$ is continuous with respect to inverse systems of compact Hausdorff pairs, and hence $(3) \Rightarrow(1)$.

Another proof, avoiding the explicit proof of continuity for $\check{F}$, easily follows from the fact that (1) and (2) are equivalent by virtue of (4.3) and (4.4). Then apply Theorem 5 to obtain the equivalence of (2) and (3).

4.5. REMARK. The example of 3.1 is continuous since $\theta$ is an equivalence but $\left(\boldsymbol{R}^{n}, \boldsymbol{R}^{n}-\{0\}\right)$ does not have the homotopy type of a triangulable pair. On the other hand, if $F$ restricted to $\mathscr{K}_{f}$ is equivalent to a functor representable by some pair $\left(E, E^{\prime}\right)$ not necessarily in $\mathscr{C}_{c}$ then $\check{F}$ is representable on $\mathscr{C}_{c}$ by a triangulable pair $(K, L)$ for which there is a singular homotopy equivalence $(K, L) \rightarrow\left(E, E^{\prime}\right)$. On the other hand, there exists group-valued functors on $\mathscr{C}_{c}$, weakly continuous, satisfying the homotopy axiom, and yet their restriction to $\mathscr{K}_{f}$ is not representable by any topological pair. As an example, we may take $F(X, A)=\check{H}^{2}(X, A ; Z) /$ torsion subgroup of $\check{H}^{2}(X, A ; Z),(X, A) \in \mathscr{C}_{c}$.

4.6. Let $(\mathscr{C}, \mathscr{K})$ be a Čech extension category where the objects of $\mathscr{C}$ are paracompact Hausdorff closed pairs as in 2.1 , and let $F: \mathscr{K} \rightarrow \mathscr{S}$ be a contravariant functor. 
THEOREM. 5. $\mathscr{F}$ is weakly continuous in $\mathscr{C}$.

Proof. Let $\left\{\left(X_{m}, A_{m}\right), i_{n, m}\right\}_{m \in M}$ be a nested system for $(X, A)$ in some fixed space $\boldsymbol{Y}$ (not necessarily in $\mathscr{C}$ ). We wish to show the map

$$
\mu: \operatorname{Dir} \operatorname{Lim} \check{F}\left(X_{m}, A_{m}\right) \rightarrow \check{F}(X, A)
$$

is bijective.

We shall first prove

4.7. LemmA. Given any barycentric map $\varphi_{\alpha}:(X, A) \rightarrow\left(X_{\alpha}, A_{\alpha}\right)$, there exists a map $f:\left(X_{n}, A_{n}\right) \rightarrow\left(X_{\alpha}, A_{\alpha}\right)$ for some index $n$ such that $f \mid(X, A) \cong \varphi_{\alpha}$.

Proof. Let $\left(V_{\alpha}, V_{\alpha}^{A}\right)$ be the indexing pair of $\alpha$. For each $v \in V_{\alpha}$ (resp., $\left.V_{\alpha}^{A}\right)$, choose an open subset $v^{\prime}$ of $Y$ with $v^{\prime} \cap X=v$, let $U$ (resp., $W$ ) be their union for all $v \in V_{\alpha}$ (resp., $V_{\alpha}^{A}$ ), and let $\alpha^{\prime}$ be the resulting covering of $(X, A)$ in $Y$. By assumption, there is an index $m$ such that $\left(X_{m}, A_{m}\right) \subset(U, W)$. Let $\alpha^{\prime \prime}=\alpha^{\prime} \mid\left(X_{m}, A_{m}\right)$, and let $\varphi_{\alpha^{*}}:\left(X_{m}, A_{m}\right) \rightarrow\left(X_{m \alpha^{*}}, A_{m \alpha^{*}}\right)$ be a barycentric map which exists by assumptions on the $\left(X_{m}, A_{m}\right)$. Note that $\varphi_{\alpha^{*}} \mid(X, A)$ maps $(X, A)$ into $\left(X_{\alpha}, A_{\alpha}\right)$ where $\left(X_{\alpha}, A_{\alpha}\right)$ is regarded as a subcomplex pair of $\left(X_{m \alpha^{*}}, A_{m \alpha^{*}}\right)$. Furthermore, $\varphi_{\alpha^{\mu}} \mid(X, A) \cong \varphi_{\alpha}$. Take the (open) regular neighborhood pair $(P, Q)$ of $\left(X_{\alpha}, A_{\alpha}\right)$ in the first barycentric subdivisions of $\left(X_{m \alpha^{*}}, A_{m \alpha^{\prime}}\right)$, and let $r:(P, Q) \rightarrow\left(X_{\alpha}, A_{\alpha}\right)$ be the canonical retraction map. Choose an index $n>m$ such that $\left(\varphi_{\alpha^{\mu}}\left(X_{n}\right), \varphi_{\alpha^{n}}\left(A_{n}\right)\right) \subset(P, Q)$. Then $r \circ\left(\varphi_{\alpha^{n}} \mid\left(X_{n}, A_{n}\right)\right)$ is the desired map $f$. This completes the proof of the lemma.

We now prove that $\mu$ is surjective. Suppose $a \in \mathscr{F}(X, A)$ is represented by some $a_{\alpha} \in F\left(X_{\alpha}, A_{\alpha}\right)=\breve{F}\left(X_{\alpha}, A_{\alpha}\right)$. Then by $2.4, \breve{F}\left(\varphi_{\alpha}\right)\left(a_{\alpha}\right)=a$. Choose $f:\left(X_{n}, A_{n}\right) \rightarrow$ $\left(X_{\alpha}, A_{\alpha}\right)$ as in 4.7. Then $\breve{F}(f)\left(a_{\alpha}\right) \in F\left(X_{n}, A_{n}\right)$ which is mapped onto $a$ by $\breve{F}\left(i_{n}\right)$ where $i_{n}:(X, A) \subset\left(X_{n}, A_{n}\right)$ is the inclusion map. This proves that $\mu$ is surjective.

To prove that $\mu$ is injective, we need

4.8. Corollary. If $\left(E, E^{\prime}\right)$ is a triangulable pair then the canonical map

$$
\operatorname{Dir} \operatorname{Lim}\left[X_{m}, A_{m} ; E, E^{\prime}\right] \stackrel{\mu^{\prime}}{\longrightarrow}\left[X, A ; E, E^{\prime}\right]
$$

is bijective.

Proof. That $\mu^{\prime}$ is surjective follows from 2.8 and what has just been proved.

Let $f, g:\left(X_{m}, A_{m}\right) \rightarrow\left(E, E^{\prime}\right)$ be maps such that $f \cong g$ on $(X, A)$. Let $h:(X, A) \times I$ $\rightarrow\left(E, E^{\prime}\right)$ be a homotopy with $h_{0}=f$ and $h_{1}=g$. Define a map

$$
H:\left(X_{m}, A_{m}\right) \times\{0\} \cup(X, A) \times I \cup\left(X_{m}, A_{m}\right) \times\{1\} \rightarrow\left(E, E^{\prime}\right)
$$

by $f \times\{0\} \cup h \cup g \times\{1\}$. Observe that $\left\{\left(X_{m}, A_{m}\right) \times\{0\} \cup\left(X_{n}, A_{n}\right) \times I \cup\left(X_{m}, A_{m}\right)\right.$ $\times\{1\}\}_{n>m}$ is a nested system for the domain of $H$ in $Y \times I$. Now apply the fact that $\mu^{\prime}$ is surjective for any nested system, and get a map $\bar{H}:\left(X_{n}, A_{n}\right) \times I \rightarrow\left(E, E^{\prime}\right)$ for some $n>m$ which is homotopic to $H$ if restricted to $\left(X_{n}, A_{n}\right) \times\{0\} \cup(X, A) \times I$ $\cup\left(X_{n}, A_{n}\right) \times\{1\}$. On $X_{n}, f=H_{0} \cong \bar{H}_{0} \cong \bar{H}_{1} \cong H_{1}=g$. This proves that $\mu^{\prime}$ is injective. 
Continuation of Proof of Theorem 5. To prove that $\mu$ is injective, let $a_{m}, b_{m}$ $\in \check{F}\left(X_{m}, A_{m}\right)$ such that $\breve{F}\left(i_{m}\right)\left(a_{m}\right)=\breve{F}\left(i_{m}\right)\left(b_{m}\right)=c \in \check{F}(X, A)$ where $i_{m}:(X, A) \subset\left(X_{m}, A_{m}\right)$ denotes the inclusion map. We wish to show $\breve{F}\left(i_{m, n}\right)\left(a_{m}\right)=\breve{F}\left(i_{m, n}\right)\left(b_{m}\right) \in F\left(X_{n}, A_{n}\right)$ for some $n>m$.

There is an $\alpha \in \operatorname{Cov}^{\mathscr{X}}\left(X_{m}, A_{m}\right)$ such that both $a_{m}$ and $b_{m}$ are represented by some $a_{m \alpha}$ and $b_{m \alpha}$ in $\check{F}\left(X_{m \alpha}, A_{m \alpha}\right)$. By $2.4, \check{F}\left(\varphi_{\alpha}\right)\left(a_{m \alpha}\right)=a_{m}$ and $\check{F}\left(\varphi_{\alpha}\right)\left(b_{m \alpha}\right)=b_{m}$ where $\varphi_{\alpha}:\left(X_{m}, A_{m}\right) \rightarrow\left(X_{m \alpha}, A_{m \alpha}\right)$ is a barycentric map. Let $\alpha^{\prime}=\alpha \mid(X, A)$. Then we have the commutative diagram

$$
\begin{gathered}
\check{F}\left(X_{m}, A_{m}\right) \stackrel{\check{F}\left(i_{m}\right)}{\longrightarrow} \check{F}(X, A) \\
\left\lceil\check{F}\left(\varphi_{\alpha}\right)\right. \\
\check{F}\left(X_{m \alpha}, A_{m \alpha}\right) \underset{\check{F}\left(i_{m \alpha}\right)}{\longrightarrow} \check{F}\left(X_{\alpha^{\prime}}, A_{\alpha^{\prime}}\right) .
\end{gathered}
$$

Let $a_{\alpha^{\prime}}=\breve{F}\left(i_{m \alpha}\right)\left(a_{m \alpha}\right)$ and $b_{\alpha^{\prime}}=\breve{F}\left(i_{m \alpha}\right)\left(b_{m \alpha}\right)$. Then $\check{F}\left(\varphi_{\alpha^{\prime}}\right)\left(a_{\alpha^{\prime}}\right)=c=\breve{F}\left(\varphi_{\alpha^{\prime}}\right)\left(b_{\alpha^{\prime}}\right)$. Hence by definition of $\breve{F}(X, A)$, there is $\beta \in \operatorname{Cov}^{\mathscr{X}}(X, A)$ such that $\beta>\alpha^{\prime}$ and $\check{F}\left(\pi_{\alpha^{\prime} \beta}\right)\left(a_{\alpha^{\prime}}\right)$ $=\check{F}\left(\pi_{\alpha^{\prime} \beta}\right)\left(b_{\alpha^{\prime}}\right)$.

By 4.7 , there is a map $f:\left(X_{m^{\prime}}, A_{m^{\prime}}\right) \rightarrow\left(X_{\alpha^{\prime}}, A_{\alpha^{\prime}}\right)$ for some $m^{\prime}>m$ such that $f \circ i_{m^{\prime}} \cong \varphi_{\alpha^{\prime}}$. We may assume $m=m^{\prime}$. By doing the same to $\beta$, we get a map $g:\left(X_{n}, A_{n}\right)$ $\rightarrow\left(X_{\beta}, A_{\beta}\right)$ for some $n>m$ such that $g \circ i_{n} \cong \varphi_{\beta}$. Consider the diagram

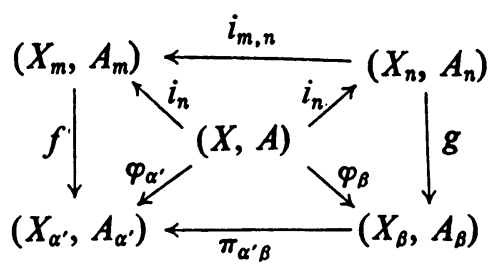

Since $f \circ i_{m} \cong \varphi_{\alpha^{\prime}} \cong \pi_{\alpha^{\prime} \beta} \circ g \circ i_{n}$, by 4.8 , there is $p>n$ such that $\pi_{\alpha^{\prime} \beta} \circ g \cong f$ on $X_{p}$, or $\pi_{\alpha^{\prime} \beta} \circ g \circ i_{n, p} \cong f \circ i_{n, p}$. Hence

$$
\begin{aligned}
\check{F}\left(i_{m, p}\right)\left(a_{m}\right) & =\breve{F}\left(i_{m, p}\right) \check{F}(f)\left(a_{\alpha^{\prime}}\right)=\check{F}\left(f \circ i_{m, p}\right)\left(a_{\alpha^{\prime}}\right) \\
& =\breve{F}\left(\pi_{\alpha^{\prime} \beta} \circ g \circ i_{n, p}\right)\left(a_{\alpha^{\prime}}\right)=\check{F}\left(g \circ i_{n, p}\right) \check{F}\left(\pi_{\alpha^{\prime} \beta}\right)\left(a_{\alpha^{\prime}}\right) .
\end{aligned}
$$

Similarly, $\check{F}\left(i_{m, p}\right)\left(b_{m}\right)=\breve{F}\left(g \circ i_{n, p}\right) \check{F}\left(\pi_{\alpha^{\prime} \beta}\right)\left(b_{\alpha^{\prime}}\right)$, but $\beta$ was taken so that $\breve{F}\left(\pi_{\alpha^{\prime} \beta}\right)\left(a_{\alpha^{\prime}}\right)$ $=\check{F}\left(\pi_{\alpha^{\prime} \beta}\right)\left(b_{\alpha^{\prime}}\right)$ and hence $\check{F}\left(i_{m, p}\right)\left(a_{m}\right)=\breve{F}\left(i_{m, p}\right)\left(b_{m}\right)$. This completes the proof of Theorem 5.

4.9. Corollary. Let $(X, A)$ be a closed pair in a metric space $Y$ (or more generally a space $Y$ whose open subspaces are paracompact $)$ and let $\left\{\left(X_{m}, A_{m}\right)\right\}_{m \in M}$ be a nested system for $(X, A)$ in $Y$. Then

$$
\operatorname{Dir} \operatorname{Lim} \check{H}^{i}\left(X_{m}, A_{m} ; G\right) \approx \check{H}^{i}(X, A ; G)
$$

where $G$ is an abelian group and $\check{H}^{i}(\quad ; G)$ is the ordinary Čech cohomology functor (cf. Godement [8, II, 4.11.1]). 
Note that $X$ is not assumed to be a closed subspace of $Y$.

4.10. Let $\mathscr{K}$ be the category of locally finite, countable, finite dimensional triangulable spaces and continuous maps and let $\mathscr{C}$ be the category of spaces which are finite dimensional separable metric spaces and maps. Then, $(\mathscr{C}, \mathscr{K})$ is a Čech extension category. Let $F: \mathscr{C} \rightarrow \mathscr{S}$ be a contravariant functor satisfying the homotopy axiom, and let $\check{F}: \mathscr{C} \rightarrow \mathscr{S}$ be the Čech extension of $F \mid \mathscr{K}$.

COROllary. $\theta: \check{F} \rightarrow F$ is an equivalence if and only if $F$ is weakly continuous in $\mathscr{C}$.

Proof. This follows from Theorem 5 and the well-known fact that any finite dimensional separable metric space $X$ can be imbedded in an euclidean space $Y$ so that the space $X$ is the intersection of a nested system consisting of open triangulable neighborhoods of $X$ in $Y$.

4.11. ReMARK (ON BUNDLE THEORY). Let $G$ be a topological group such that a classifying space $B_{G}$ for principal $G$-bundles over paracompact Hausdorff spaces has the homotopy type of a $C W$-complex. Let $X$ be an arbitrary paracompact subspace of a Hausdorff space $Y$ and let $\left\{U_{m}\right\}_{m \in M}$ be a nested system of paracompact subspaces of $Y$ for $X$. If $\xi$ is a principal $G$-bundle on $X$ then there exists a principal $G$-bundle $\xi^{\prime}$ on some $U_{m}$ such that $\xi^{\prime} \mid X$ is equivalent to $\xi$.

Furthermore, if $\xi_{1}$ and $\xi_{2}$ are principal $G$-bundles over $U_{m}$ so that $\xi_{1} \mid X$ and $\xi_{2} \mid X$ are equivalent, then $\xi_{1} \mid U_{n}$ and $\xi_{2} \mid U_{n}$ are already equivalent for some $n>m$.

\section{Miscellaneous remarks.}

5.1. There are other basic properties of the classical Čech cohomology theory. For example, the excision axiom and the exactness axiom. Since the latter is concerned with more than one functor, we do not consider it specifically here. However, suitably defined generalized Čech cohomology theory will satisfy this axiom, and in fact, the proof given by Eilenberg and Steenrod is valid for the generalized theory.

5.2. Let $F$ be a contravariant functor with values in $\mathscr{S}$ and defined on a category of topological pairs and continuous maps. We recall the

Excision Axiom. If $(X, A) \in \mathscr{C}$ and $U$ is an open subset of $X$ with the closure of $U$ contained within the interior of $A$, then the map $F(i): F(X, A) \rightarrow$ $F(X-U, A-U)$ is bijective where $i:(X-U, A-U) \subset(X, A)$ is the inclusion map.

Some contravariant functors occurring in practice actually satisfy a stronger version of this axiom:

Strong Excision Axiom. If $(X, A) \in \mathscr{C}$ then the map $F(q): F(X|A, A| A) \rightarrow$ $F(X, A)$ is bijective where $q:(X, A) \rightarrow(X / A, A / A)$ is the quotient map.

Now let $(\mathscr{C}, \mathscr{K})$ be an abstract Čech extension category as defined in $\S 1$, and let $F: \mathscr{K} \rightarrow \mathscr{S}$ be a contravariant functor satisfying the homotopy axiom, and $\check{F}: \mathscr{C} \rightarrow \mathscr{S}$ its Čech extension.

REMARK. If $F$ satisfies the excision axiom on $\mathscr{K}$ then $F$ satisfies the strong excision axiom provided that $\mathscr{K}$ satisfies $(K, L) \in \mathscr{K} \Rightarrow(K / L, L / L) \in \mathscr{K}$. 
This is an easy consequence of the homotopy and excision axioms and is left to the reader.

THEOREM 6. If $F$ satisfies the excision axiom on $\mathscr{K}$ then so does $\breve{F}$ on $\mathscr{C}$. If $F$ is homotopy representable by a topological pair $\left(E, E^{\prime}\right)$ with $E^{\prime}$ consisting of a single point then $\check{F}$ satisfies the strong excision axiom.

The first assertion can be proved by following the proof for the corresponding theorem as given by Eilenberg-Steenrod [7, p. 243], and by making necessary modifications.

In order to prove the second assertion observe that the natural identification map $\eta:(X, A) \rightarrow(X|A, A| A)$ induces a continuous one-to-one onto map,

$$
\eta^{*}:(E, p t)^{(x / A, A / A)} \rightarrow(E, p t)^{(X, A)}
$$

Because the unit interval $I$ is compact, the natural map $\eta \times 1:(X, A) \times I \rightarrow$ $(X / A \times I, A / A \times I)$ is a quotient map. (This is a theorem of J. H. C. Whitehead and can be found in Dugundji's book, Topology, p. 262.) Thus, $(\eta \times 1)^{*}$ is also continuous, one-to-one onto, and induces a bijection of $[X \mid A, A / A ; E, p t]$ onto $[X, A ; E, p t]$. Now apply Theorem 3 .

5.3. Let $(\mathscr{C}, \mathscr{K})$ be a Čech extension category, and let $\mathscr{K}_{*}$ (respectively, $\mathscr{C}_{*}$ ) be the full subcategory of $\mathscr{K}$ (respectively, $\mathscr{C}$ ) of the based spaces, that is, the pairs $(X, A)$ where $A$ is a single point. We may define $\breve{F}_{*}$ on $\mathscr{C}_{*}$ using $F_{*}$ so that all our theorems are valid for $\breve{F}_{*}$. In order to do this, we make an added requirement that if $(K, L) \in \mathscr{K}$ then $(K / L, L / L) \in \mathscr{K}$, and define $F$ over $\mathscr{K}$ by $F(K, L)=F_{*}(K / L, L / L)$, where $F_{*}$ satisfies the homotopy axiom on $\mathscr{K}_{*}$.

Since homotopies between closed pairs of $\mathscr{C}$ induce homotopies between the corresponding pairs of $\mathscr{C}_{*}$ by the same result of J. H. C. Whitehead referred to in 5.2, $F$ satisfies the homotopy axiom on $\mathscr{K}$. By the Čech method, define $\breve{F}$ on $\mathscr{C}$, and let $\breve{F}_{*}$ be the restriction $\breve{F} \mid \mathscr{C}_{*}$. Now one can reformulate, in terms of based spaces, all our theorems and corollaries for $F_{*}$ and $\breve{F}_{*}$. The essential idea of validating this reformulation is to use the theorems for pairs as already given here and to apply the Whitehead result to the pertinent quotient spaces. We leave these obvious reformulations and details to the reader.

Appendix 1. The Maximal and Minimal Extensions.

Let $\mathscr{C}$ be an abstract category and $\mathscr{K}$ a full subcategory of $\mathscr{C}$. Denote by $\mathscr{S}$ the category of sets and set maps. Let $F_{0}: \mathscr{K} \rightarrow \mathscr{S}$ be a functor which may be covariant or contravariant. We define the category of extensions of $F_{0},\left(\mathscr{S}^{\mathcal{S}}, F_{0}\right)$ by notation, to be the category of functors $F: \mathscr{C} \rightarrow \mathscr{S}$ with the same variance as $F_{0}$ whose restrictions to $\mathscr{K}$ is identical with $F_{0}$. The morphisms in this category are defined to be all natural transformations between such functors whose restriction on $\mathscr{K}$ are the identity transformations. An initial (respectively, terminal) object in $\left(\mathscr{S}^{8}, F_{0}\right)$ is called a maximal (respectively, minimal) extension of $F_{0}$. (See Saunders Mac Lane, 
Categorical algebra, Bull. Amer. Math. Soc. 71 (1965), p. 42, for the standard definitions of initial and terminal objects.) Clearly such objects are unique up to an equivalence.

A.1.1. REMARK. Let $(\mathscr{C}, \mathscr{K})$ be a Čech extension category where the objects of $\mathscr{C}$ are paracompact Hausdorff closed pairs, and let $F_{0}: \mathscr{K} \rightarrow \mathscr{S}$ be a contravariant (respectively, covariant) functor satisfying the homotopy axiom. Then $\check{F}_{0}$ is a maximal (respectively, minimal) extension of $F_{0}$ satisfying the homotopy axiom.

To be more precise, the definitions of $\breve{F}_{0}$ should be slightly modified so that $\check{F}_{0}$ restricted to $\mathscr{K}$ is strictly identical with $F_{0}$. Now the conclusion follows from 2.4 (its analogue in the covariant case), and by passing to the category with the same objects as $\mathscr{C}$ but with the homotopy classes of maps instead of continuous maps between the topological pairs of $\mathscr{C}$.

A.1.2. Consider $\left(\mathscr{C}_{1}, \mathscr{K}_{1}\right)$ (see $\left.\S 1\right)$. Let $F_{0}: \mathscr{K}_{1} \rightarrow \mathscr{S}$ be a functor satisfying the homotopy axiom. We shall define the singular extension $F_{0}^{s}: \mathscr{C}_{1} \rightarrow \mathscr{S}$ of $F_{0}$. First we need to recall the geometric realization $R S(X, A)=(|S X|,|S A|)$ (as defined by Giever-Hu) of the singular complex over any topological pair $(X, A)$. In fact, $R S: \mathscr{C}_{1} \rightarrow \mathscr{K}_{1}$ is a covariant functor preserving the homotopy relation, and there is the natural transformation $i: R S \rightarrow 1_{\mathscr{C}_{1}}$ where $1_{\mathscr{C}_{1}}$ is the identity functor of $\mathscr{C}_{1}$ into itself. Now define $F_{0}^{s}=F_{0} \circ R S$ the composite functor. Then $F_{0}^{s}$ satisfies the homotopy axiom and $F_{0}^{s} \mid \mathscr{K}$ is naturally equivalent to $F_{0}$. Again by a slight modification, we can identify $F_{0}^{s} \mid \mathscr{K}$ with $F_{0}$ so that $F_{0}^{s}$ is an extension in the strict sense.

REMARK. If $F_{0}: \mathscr{K}_{1} \rightarrow \mathscr{S}$ is a covariant (respectively, contravariant) functor satisfying the homotopy axiom then $F_{0}^{s}: \mathscr{C}_{1} \rightarrow \mathscr{S}$ is the maximal (minimal) extension of $F_{0}$ satisfying the homotopy axiom.

The proof is not difficult and left to the reader.

A.1.3. We now describe another useful topological example of maximal and minimal extensions. Let $(\mathscr{C}, \mathscr{K})$ be $\left(\mathscr{K}_{1}, \mathscr{K}_{f}\right)$ and $F_{0}: \mathscr{K}_{f} \rightarrow \mathscr{S}$ be a functor which satisfies the homotopy axiom. For each $(X, A) \in \mathscr{K}_{1}$, the family of finitely triangulable pairs $\left\{\left(X_{\alpha}, A_{\alpha}\right) ; i_{\alpha \beta}\right\}$ forms a directed system of sets. It is directed by inclusion maps. If $F_{0}$ is covariant (respectively, contravariant) then

$\operatorname{Dir} \operatorname{Lim}\left\{F_{0}\left(X_{\alpha}, A_{\alpha}\right) ; F_{0}\left(i_{\alpha \beta}\right)\right\}$ (respectively, $\left.\operatorname{Inv} \operatorname{Lim}\left\{F_{0}\left(X_{\alpha}, A_{\alpha}\right) ; F_{0}\left(i_{\alpha \beta}\right)\right\}\right)$

defines the maximal covariant (respectively, minimal contravariant) extension of $F_{0}$ among the extensions which satisfy the homotopy axiom. This corresponds to homology (respectively, cohomology) with compact carriers [7, p. 255]. Similar examples can be described using $\mathscr{K}=\mathscr{K}_{f d}$ and $\mathscr{C}=\mathscr{K}_{1}$, or $\mathscr{K}=\mathscr{C}_{c}$ and $\mathscr{C}=$ category of Hausdorff pairs.

A.1.4. Let $F, F_{1} \in\left(\mathscr{S}^{\mathscr{C}}, F_{0}\right)$, where $F_{1}$ is a maximal (respectively, minimal) extension of $F_{0}$. Suppose that $\theta$ is the unique natural transformation $\theta: F_{1} \rightarrow F$ (respectively, $\theta: F \rightarrow F_{1}$ ) and that there exists a natural transformation $\psi: F \rightarrow F_{1}$ (respectively, $\psi: F_{1} \rightarrow F$ ) with $\psi$ the identity on $\mathscr{K}$. Then, $\psi \theta=1$ (respectively, 
$\theta \psi=1)$. This fact is an immediate consequence of the definition of initial and terminal objects. We use it now in proving an abstract form of a weak version of Theorem 2.

Proposition. Let $F, F_{1} \in\left(\mathscr{S}^{\mathscr{C}}, F_{0}\right)$, where $F_{1}$ is a maximal extension of $F_{0}$, and $F$ is homotopy representable by an object $E$ in $\mathscr{C}$. If $E$ is dominated by an object $E^{\prime}$ in $\mathscr{K}$, (that is, if there exist morphisms $f: E \rightarrow E^{\prime}, g: E^{\prime} \rightarrow E$, such that $F(g \circ f)=F(1)$ ), then $\theta$, the unique natural transformation between $F$ and $F_{1}$, is bijective.

Proof. Define $e \in F_{1}(E)$ by $F(g) \theta_{E^{\prime}}^{-1} F(f)(1)$, in the covariant case, and $F_{1}(f) \theta_{E^{\prime}}^{-1} F(g)(1)$, in the contravariant case. As in 2.5, define the natural transformation $\psi^{e}: F \rightarrow F_{1}$ such that $\theta \psi^{e}=1$. We check this in the covariant case: $\theta_{x} \psi_{x}^{e}(h)=$ $\theta_{x} F_{1}(h)(e)=F(h) \theta_{\Sigma}(e)=F(h) \theta_{\Sigma}\left(F_{1}(g) \theta_{\bar{E}^{\prime}}^{-1} F(f)(1)\right)=F(h) F(g \circ f)(1)=h$, for $h \in F(X)$, and $X \in \mathscr{C}$. The contravariant case is similar.

Since $\theta$ restricted to $\mathscr{K}$ is the identity, it follows, from above, that $\psi^{e}$ is also the identity on $\mathscr{K}$. Therefore, $\psi \theta=1$, and consequently, $\theta$ is an equivalence. This completes the proof.

We give a topological application which is the analogue of Theorem 2 for the singular extension. It is useful to restrict ourselves to functors and extensions which satisfy the homotopy axiom.

CoROllaRY 1. Let $\left(\mathscr{C}_{1}, \mathscr{K}_{1}\right)$ be as in $\S 1$, and $F_{0}$ a covariant set-valued functor from $\mathscr{K}_{1}$ which satisfies the homotopy axiom. Suppose there exists $F \in\left(\mathscr{S}^{\mathscr{C}_{1}}, F_{0}\right)$, which is homotopy representable by a pair $\left(E, E^{\prime}\right)$. Then, $\theta: F_{0}^{s} \rightarrow F_{1}$ is an equivalence if and only if $\left(E, E^{\prime}\right)$ has the homotopy type of a triangulable pair.

Proof. By A.1.2 $F_{0}^{s}$ can be regarded as a maximal extension of $F_{0}$. If $\left(E, E^{\prime}\right)$ has the homotopy type of a triangulable pair then the proposition implies that $\theta$ is an equivalence. On the other hand, if $\theta$ is an equivalence, it is easy to see that $\left(E, E^{\prime}\right)$ has the homotopy type of a triangulable pair, because the map $e=\theta_{\left(E, E^{\prime}\right)}^{-1}(1):\left(E, E^{\prime}\right)$ $\rightarrow\left(|S E|,\left|S E^{\prime}\right|\right)$, and the realization map $\left(|S E|,\left|S E^{\prime}\right|\right) \rightarrow\left(E, E^{\prime}\right)$ define a dominance of $\left(E, E^{\prime}\right)$ by $\left(|S E|,\left|S E^{\prime}\right|\right)$.

Corollary 2. Let $\left(E, E^{\prime}\right)$ be a triangulable pair and $\left\{\left(E_{\alpha}, E_{\alpha}^{\prime}\right)\right\}$ the family of finitely triangulable subpairs of $\left(E, E^{\prime}\right)$. Then the natural map

$$
\operatorname{Dir} \operatorname{Lim}_{\alpha}\left[E, E^{\prime} ; E_{\alpha}, E_{\alpha}^{\prime}\right] \rightarrow\left[E, E^{\prime} ; E, E^{\prime}\right]
$$

is bijective if and only if $\left(E, E^{\prime}\right)$ is dominated by a finitely triangulable pair.

Proof. Set $F_{0}=\left[E, E^{\prime} ; \quad\right]$ on $K_{f}$. Extend to $F_{0}^{m}$ on $\mathscr{K}_{1}$ by defining $F_{0}^{m}(X, A)$ $=\operatorname{Dir} \operatorname{Lim}_{\alpha}\left[E, E^{\prime}, X_{\alpha}, A_{\alpha}\right]$ for each $(X, A) \in \mathscr{K}_{1}$, where $\left\{\left(X_{\alpha}, A_{\alpha}\right)\right\}$ denotes the family of triangulable subpairs of $(X, A) . F_{0}^{m}$ is a maximal extension of $F_{0}$ which satisfies the homotopy axiom, (A.1.3). The functor $F=\left[E, E^{\prime} ; \quad\right]$ defined on $\mathscr{K}_{1}$ is also an extension of $F_{0}$ which satisfies the homotopy axiom. By the proposition, if $\left(E, E^{\prime}\right)$ is dominated by a finitely triangulable pair, then $\theta: F_{0}^{m} \rightarrow F$ is bijective. On 
the other hand, if $\theta_{E, E^{\prime}}: F_{0}^{m} \rightarrow F$ is bijective, then there exists $e \in F_{0}^{m}\left(E, E^{\prime}\right)$ such that $\theta_{E, E^{\prime}}(e)$ is the homotopy class represented by $1_{E, E^{\prime}}:\left(E, E^{\prime}\right) \rightarrow\left(E, E^{\prime}\right)$. Since $F_{0}^{m}$ has compact carriers, there exists $\left(E_{\alpha}, E_{\alpha}^{\prime}\right) \in \mathscr{K}_{f}$ and maps $e_{\alpha}:\left(E, E^{\prime}\right) \rightarrow\left(E_{\alpha}, E_{\alpha}^{\prime}\right)$ such that $i_{\alpha} \circ e_{\alpha}:\left(E, E^{\prime}\right) \rightarrow\left(E_{\alpha}, E_{\alpha}^{\prime}\right) \rightarrow\left(E, E^{\prime}\right)$ represents $e$. That is $i_{\alpha} \circ e_{\alpha}$ is homotopic to the identity.

We point out that we have shown considerably more than we have stated in Corollary 2. Similar statements can be made with different directed systems such as those mentioned at the end of A.1.3.

A.1.5. At the end of $\S 1$ we defined two covariant functors

$$
\text { Čech: } \mathscr{S}^{\mathscr{X}} \rightleftarrows \mathscr{S}^{\mathscr{C}}: \text { Res }
$$

where $(\mathscr{C}, \mathscr{K})$ is a Čech extension category. It follows from 2.2 that Res $\circ$ Čech $F$ may be identified with $F$ for each functor in $\mathscr{S} \mathscr{X}$. Assume further that the objects of $\mathscr{C}$ are paracompact closed pairs so that $\theta_{F}$ : Čech $\circ \operatorname{Res} F \rightarrow F$ is well defined for functors $F$ in $\mathscr{S}^{\mathcal{Y}}$. Let $F$ and $G$ be functors in $\mathscr{S}^{\mathscr{X}}$ and $\mathscr{S}^{\mathscr{C}}$ respectively. For each natural transformation $\eta$ : Čech $F \rightarrow G$ define $R(\eta)$ : Res Čech $F=F \rightarrow \operatorname{Res} G$ by restriction, and for each natural transformation $\xi: F \rightarrow \operatorname{Res} G$ define $C(\xi)$ to be the composition of Čech $F \stackrel{\text { ¿ech }}{\longrightarrow}$ Čech $\operatorname{Res} G \stackrel{\theta_{G}}{\longrightarrow} G$. It follows from 2.2 and 2.4 that $C R(\eta)=\eta$ and $R C(\xi)=\xi$. This yields a natural equivalence between

$$
\operatorname{Morph}_{\mathscr{S} \mathscr{B}}(\check{C ̆ e c h} F, G) \rightarrow \operatorname{Morph}_{\mathscr{S}} \mathscr{X}(F, \operatorname{Res} G) .
$$

In other words, the Čech extension functor Čech is the adjoint of the restriction functor Res in the usual sense (generalized to larger categories than "legitimate" ones. The categories $\mathscr{S}^{\mathscr{C}}$ and $\mathscr{S}^{\mathscr{X}}$ will be legitimate if we demand $\mathscr{K}$ and $\mathscr{C}$ to be small categories).

We leave it to the reader to formulate a similar statement for the singular extension functor defined on a category of covariant functors to the effect that the singular extension functor is the adjoint of the corresponding restriction functor Res. Dually, for the appropriate extension categories, one can deduce that the Cech extension functor, for covariant functors, and the singular extension functor, for contravariant functors, are coadjoints of the restriction functor Res.

Appendix 2. The Dominance Extension Category.

Let $\mathscr{C}$ be an abstract category. An object $X$ is said to be dominated by an object $Y$ if there is a sequence: $X \stackrel{f}{\rightarrow} Y \stackrel{g}{\rightarrow} X$ with $g f=1_{X}$. Let $\mathscr{C}_{0}$ be a full subcategory of $\mathscr{C}$, and let $\mathscr{D}=\mathscr{D}\left(\mathscr{C}_{0}\right)$ be the full subcategory of $\mathscr{C}$ whose objects are dominated by objects of $\mathscr{C}_{0}$.

THEOREM. If $F: \mathscr{C}_{0} \rightarrow \mathscr{S}$ is a contravariant functor there exists a unique extension (up to an equivalence) to a contravariant functor: $\mathscr{D} \rightarrow \mathscr{S}$. If $F^{\prime}$ and $F^{\prime \prime}$ are contravariant functors: $\mathscr{D} \rightarrow \mathscr{S}$ then any natural transformation: $F^{\prime}\left|\mathscr{C}_{0} \rightarrow F^{\prime \prime}\right| \mathscr{C}_{0}$ admits a unique extension to a natural transformation: $F^{\prime} \rightarrow F^{\prime \prime}$. In particular, if $F^{\prime} \mid \mathscr{C}_{0}$ $\rightarrow F^{\prime \prime} \mid \mathscr{C}_{0}$ is an equivalence, then the extension is an equivalence. 
REMARK. For topological applications, one needs to consider categories in which objects are topological pairs as before (or more generally $n$-ads, $n \geqq 1$ ) but morphisms are homotopy classes of maps instead of just continuous maps between the objects.

As an illustration of a topological application consider $F: \mathscr{K}_{f} \rightarrow \mathscr{S}$ defined as in $\$ 1$ and satisfying the homotopy axiom. Then there exists a uniquely defined (up to equivalence) extension of $F$ to all spaces dominated by finitely triangulable pairs, and homotopy classes of maps. For example, the functor $F$ automatically extends to compact ANR's since they are dominated by finitely triangulable pairs.

Another illustration is that if $(\mathscr{C}, \mathscr{K})$ is a Čech extension category then all extensions of $F: \mathscr{K} \rightarrow \mathscr{S}$ to $\mathscr{D}(\mathscr{K})$ are equivalent to $\breve{F}$ on $\mathscr{D}(\mathscr{K}) \mid(\mathscr{D}(\mathscr{K}) \cap \mathscr{C})$. For example, the classical axioms for ordinary cohomology on finitely triangulable pairs constitute a uniqueness category. This uniqueness category is known to be extendable to the category of spaces having the homotopy type of finite $C W$-pairs. The dominance category, on the other hand, is strictly larger than this category and is a uniqueness category.

Proof of the Theorem. Let $\tilde{D}$ be the category in which the objects are sequences $X \stackrel{f}{\rightarrow} Y \stackrel{g}{\rightarrow} X$ with $Y \in \mathscr{C}_{0}$ and $g f=1_{X}$, and if $S=(X \rightarrow \cdots)$ and $S^{\prime}=\left(X^{\prime} \rightarrow \cdots\right)$ are such sequences then the morphisms $S \rightarrow S^{\prime}$ are precisely those: $X \rightarrow X^{\prime}$. In what follows, it is often convenient to look at the commutative diagram

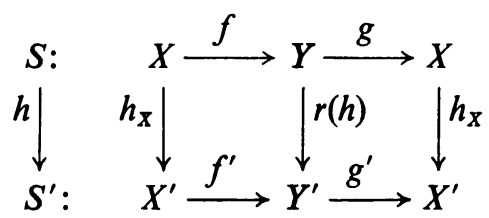

where $r(h)=f^{\prime} h_{X} g$. In fact, if we let $r(S)=Y$ then we get a covariant functor $r: \tilde{D}$ $\rightarrow \mathscr{C}_{0}$ so that $F \circ r: \tilde{\mathscr{D}} \rightarrow \mathscr{S}$ is a contravariant functor.

Define a new contravariant functor $\tilde{F}: \tilde{\mathscr{D}} \rightarrow \mathscr{S}$ by: if $S \in \tilde{\mathscr{D}}$ then $\tilde{F}(S)=$ Image $F(f g) \subset F(Y)$, and if $h: S \rightarrow S^{\prime}$ is in $\tilde{D}$ then $\tilde{F}(h)=(F r)(h)$ restricted to the subset $\tilde{F}\left(S^{\prime}\right)$ of $F\left(Y^{\prime}\right)$. We need first show $\tilde{F}(h)\left(\tilde{F}\left(S^{\prime}\right)\right) \subset \tilde{F}(S)$. Observe $F(f g) F r(h)$ $=F r(h)=F r(h) F\left(f^{\prime} g^{\prime}\right)$. Let $u \in \operatorname{Image} F\left(f^{\prime} g^{\prime}\right)$, that is, $u=F\left(f^{\prime} g^{\prime}\right)(v)$ for some $v \in$ $F\left(Y^{\prime}\right)$. Applying $F r(h)$, we get $F r(h)(u)=F r(h) F\left(f^{\prime} g^{\prime}\right)(v)=F r(h)(v)=F(f g)(F r)(h)(v)$ $\in$ Image $F(f g)=\tilde{F}(S)$. It is now clear that we get the contravariant functor $\tilde{F}: \mathscr{D} \rightarrow \mathscr{S}$. In particular, if $h$ is a morphism in $\mathscr{D}$ which is invertible then so is $\tilde{F}(h)$ and $\widetilde{F}(h)$ is a bijection. It follows that for each object $X$ in $\mathscr{D}$, the system $\mathscr{F}(X)$ $=\{\tilde{F}(S), \tilde{F}(h)\}$ where the first object of the sequence $S$ is $X$ and $h_{X}=1_{X}$ is a transitive system. (See [7, p. 17] for definition of transitive system.) Let $F^{\prime}(X)$ equal the transitive limit of $\mathscr{F}(X)$. If $k: X \rightarrow X^{\prime}$ is a morphism in $\mathscr{D}$ then by considering all $h: S \rightarrow S^{\prime}$ in $\mathscr{D}$ with $h_{X}=k$, we get the canonical map $\mathscr{F}(k): \mathscr{F}\left(X^{\prime}\right) \rightarrow \mathscr{F}(X)$ of the transitive system. This then induces a map: $F^{\prime}(k): F^{\prime}\left(X^{\prime}\right) \rightarrow F^{\prime}(X)$. The functoriality of $F^{\prime}$ is easy to check, and we get the contravariant functor 
$F^{\prime}: \mathscr{D} \rightarrow \mathscr{S}$. If $X$ is an object $\in \mathscr{C}_{0}$ then $S_{X}=\left(X^{1 X} \rightarrow X^{1 X} \rightarrow X\right)$ is an object $\in \tilde{\mathscr{D}}$ and $\tilde{F}\left(S_{X}\right)=F(X)$. Hence $F^{\prime} \mid \mathscr{C}_{0}$ is naturally equivalent to $F$. This gives us the existence of an extension of $F$. Since the uniqueness of an extension follows from the second half of the theorem, it suffices to prove the second half.

For any object $X \in \mathscr{D}$, choose a sequence $S=(X \stackrel{f}{\rightarrow} Y \stackrel{g}{\rightarrow} X) \in \tilde{\mathscr{D}}$, and define $\eta_{X}^{\prime}: F^{\prime}(X) \rightarrow F^{\prime \prime}(X)$ by $\eta_{X}^{\prime}=F^{\prime \prime}(f) \eta_{Y} F^{\prime}(g)$ where $\eta$ is a given natural transformation: $F^{\prime}\left|\mathscr{C}_{0} \rightarrow F^{\prime \prime}\right| \mathscr{C}_{0}$. We leave it to the reader to verify that $\eta_{X}$ does not depend on the choice of such sequence $S$ and that $\eta^{\prime}$ is natural with respect to the morphisms in $\mathscr{D}$, and it is the only possible extension of $\eta$. This completes the proof.

We remark finally that the assumption of contravariance is only an assumption for definiteness. The corresponding results of this Appendix are valid for covariant functors $\left({ }^{4}\right)$.

\section{BIBLIOGRAPHY}

1. M. F. Atiyah, Thom complexes, Proc. London Math. Soc. 11 (1961), 291-310.

2. M. F. Atiyah and F. Hirzebruch, Vector bundles and homogeneous spaces, pp. 7-38, Proc. Sympos. Pure Math., Vol. 3, Amer. Math. Soc., Providence, R. I., 1961.

3. M. G. Barratt, Track groups. II, Proc. London Math. Soc. 5 (1955), 285-329.

4. E. H. Brown, Jr., Cohomology theories, Ann. of Math. 75 (1962), 467-484.

5. P. E. Conner and E. E. Floyd, Differentiable periodic maps, Springer, Berlin, 1964.

6. A. Dold, Relations between ordinary and extraordinary homology, pp. 2-9, Colloq. on Algebraic Topology, Aarhus, 1962.

7. S. Eilenberg and N. Steenrod, Foundations of algebraic topology, Princeton Univ. Press, Princeton, N. J., 1952.

8. R. Godement, Topologie algébrique et théorie des faisceaux, Actualitiés Sci. Ind., No. 1252, Hermann, Paris, 1958.

9. S. T. Hu, Mappings of a normal space into an absolute neighborhood retract, Trans. Amer. Math. Soc. 64 (1948), 336-358.

10. P. Huber, Homotopical cohomology and Čech cohomology, Math. Ann. 144 (1961), 73-76.

11. J. W. Milnor, On spaces having the homotopy type of a CW-complex, Trans. Amer. Math. Soc. 90 (1959), 272-280.

12. - Microbundles. I, Topology 3 (1964), suppl. 1, 53-80.

13. E. H. Spanier, Algebraic topology, McGraw-Hill, New York, 1966.

14. —_ Borsuk's cohomotopy groups, Ann. of Math. 50 (1949), 203-245.

15. G. W. Whitehead, Generalized homology theories, Trans. Amer. Math. Soc. 102 (1962), 227-283.

UNIVERSITY OF MICHIGAN, ANn Arbor, Michigan

INSTITUTE FOR ADVANCED STUDY,

Princeton, NeW Jersey

(4) We learned from the referee that the range category does not have to be the set category $\mathscr{S}$; it suffices that $(1, p)$ has a difference kernel for every idempotent morphism $p$. 